\title{
Pierre Salama, Migrants et lutte contre les discriminations en Europe
}

Strasbourg, Éditions du Conseil de l'Europe, 2010, 101 p.

Katerina Seraïdari

\section{(2) OpenEdition}

\section{Journals}

Édition électronique

URL : http://journals.openedition.org/assr/23565

DOI : $10.4000 /$ assr.23565

ISSN : $1777-5825$

Éditeur

Éditions de l'EHESS

Édition imprimée

Date de publication : 31 décembre 2011

Pagination : 270

ISBN : 9782713223273

ISSN : 0335-5985

Référence électronique

Katerina Seraïdari, « Pierre Salama, Migrants et lutte contre les discriminations en Europe », Archives de sciences sociales des religions [En ligne], 156 | octobre-décembre 2011, document 156-101, mis en ligne le 17 février 2012, consulté le 21 septembre 2020. URL : http://journals.openedition.org/assr/ 23565 ; DOI : https://doi.org/10.4000/assr.23565

Ce document a été généré automatiquement le 21 septembre 2020

(C) Archives de sciences sociales des religions 


\title{
Pierre Salama, Migrants et lutte contre les discriminations en Europe
}

Strasbourg, Éditions du Conseil de l'Europe, 2010, 101 p.

\author{
Katerina Seraïdari
}

\section{RÉFÉRENCE}

Pierre SALAMA, Migrants et lutte contre les discriminations en Europe, Strasbourg, Éditions du Conseil de l'Europe, 2010, $101 \mathrm{p}$.

1 En s'appuyant sur les recommandations du Livre blanc sur le dialogue interculturel "Vivre ensemble dans l'égale dignité» du Conseil de l'Europe (2008), cet ouvrage propose une synthèse des questions sociétales touchant aux migrants. La première partie offre un panorama de l'immigration. Les immigrés (appelés aussi dans le texte "primoimmigrants") sont définis comme des personnes nées à l'étranger (foreign born) et n'ayant pas la nationalité du pays d'accueil à la naissance (p. 12). Les flux d'immigration, difficiles à mesurer, sont différenciés des «stocks » représentant les communautés étrangères déjà installées légalement dans le pays d'accueil (migrants résidents), et dont la taille peut être évaluée de manière fiable (p. 21-22). Tout au long du livre, l'auteur examine les "politiques dites "d'accommodement raisonnable" de type " bottom up" "(p.9). Quand les États demandent aux candidats à l'immigration de «démontrer» leur désir de s'intégrer, la «charge de la preuve» est inversée : la manifestation du désir précède et conditionne l'aide accordée et la mise en place d'initiatives étatiques visant à l'intégration (p.22-23; aussi p. 34). L'auteur désapprouve cette demande de réciprocité qui va à l'encontre des recommandations du Livre blanc. De même, la politique de l'immigration «choisie » est critiquée puisqu'elle favorise « la fuite des cerveaux ». 
2 Dans la deuxième partie, les politiques d'intégration de différents pays d'Europe sont évaluées, en fonction de leurs capacités à diminuer les discriminations économiques existantes. En fait, cette partie est dédiée aux discriminations que les immigrés et leurs descendants subissent: sur le marché de travail (ce qui empêche l'intégration par le travail et qui conduit au déclassement les immigrés hautement qualifiés) et sur le plan résidentiel (ségrégation urbaine, également liée à la ségrégation scolaire). Le terme «élève allochtone (ou de première génération)» se juxtapose ici à celui de l'« élève autochtone" (p. 48-49). L'auteur rappelle que le Conseil de l'Europe encourage la scolarisation des enfants « dans leur variété linguistique maternelle »; néanmoins, cela «ne doit pas se faire au détriment de la langue du pays d'accueil» (p. 52). Dans cette partie, l'auteur distingue les "enfants allochtones", d'un côté, et les "enfants d'immigrés » (p.55), de l'autre. Les enfants d'immigrés (ou issus de l'immigration) seraient-ils une catégorie intermédiaire entre les allochtones (nés à l'étranger) et les autochtones, qui ne peut être appréhendée que par la référence à des liens familiaux nécessairement déterritorialisés? Nous retrouvons ici le déterminisme social de catégories comme foreign born et native born. Un immigré foreign born ne peut jamais sortir de cette catégorisation, même s'il est naturalisé ou s'il devient migrant résident ; de même, ses enfants ne peuvent pas devenir des autochtones, même s'ils sont native born. Après combien de générations quelqu'un "issu de l'immigration" devient-il autochtone, et qui décide de l'attribution de ce titre, qui n'a apparemment rien à avoir avec la naturalisation ? Y aurait-il donc trois catégories de citoyens, les autochtones, les foreign born (dans le cas des migrants résidents naturalisés) et les native born? L'auteur utilise ces termes sans réellement les questionner, même s'il reconnaît que la vulnérabilité de ces populations dépend de leur statut (l'opposition entre légaux et illégaux étant également prise en compte). Mais ces statuts ne sont pas «naturels », ce sont des catégorisations qui ont un impact réel sur le vécu des individus. Le lecteur aurait attendu une réflexion plus poussée sur ces catégories séparant les enfants des autochtones et des allochtones (appartenant à une terre, à une patrie) des enfants des immigrés (n'appartenant qu'à leurs propres parents et leurs semblables).

3 La troisième partie commence par interroger précisément le sens de mots dont la signification change «selon l'histoire propre à chacun des pays et les contextes historiques» (p.61). P. Salama propose l'utilisation du terme «inclusion » au lieu de celui d'intégration. Il examine par la suite les différentes politiques étatiques vis-à-vis de la diversité culturelle, tout en soulignant l'importance du principe d'interculturalité. Celle-ci "cherche à promouvoir la mixité ethnique dans l'habitat", préconise la réalisation de statistiques ethniques et de campagnes de promotion de la convivialité interculturelle (p.64-65). Afin de prouver l'efficacité de cette politique et l'échec du modèle communautariste (qui peut institutionnaliser des séparations au sein de la société), P. Salama donne comme exemple le cas des musulmans britanniques qui, selon un sondage de 2009, "sont beaucoup plus conservateurs que les musulmans français ou allemands pour tout ce qui concerne les relations sexuelles avant le mariage ", mais aussi l'homosexualité et l'avortement (p. 66-67). Il revient ici à la notion d'« accommodement raisonnable » qui peut résoudre le conflit entre deux droits (par exemple, le droit de pratiquer sa religion et l'égalité entre genres). Il s'oppose à la tendance de limiter le dialogue interculturel « à la reconnaissance des arts propres aux populations immigrées (art culinaire, folklore, chants)» (p. 70) - position que d'autres études récentes, comme celles éditées par Steven Vertovec et Susanne Wessendorf (The Multicultural Backlash. European Discourses, Policies and Practices, London and New York, 
Routledge, 2010), adoptent. Car la reconnaissance de la diversité est facilement acceptée par les élites, étant donné qu'elle ne met pas en question l'existence d'inégalités sociales et économiques (p. 82-83). La question de la globalisation et de la déterritorialisation des cultures y est aussi traitée. En ce qui concerne la part de la religion dans l'éducation interculturelle, il propose «d'enseigner dans les écoles l'histoire des religions, des faits religieux mais aussi de l'athéisme » (p. 74) : «si "ne pas croire" n'est plus enseigné dans les écoles, alors le religieux peut paraître comme naturel et miner les fondements de la séparation des Églises et de l'État » (p. 76).

Dans un dernier temps, l'auteur examine l'efficacité de la discrimination positive (affirmative actions), en montrant successivement les thèses de ceux qui la critiquent et de ceux qui la défendent. Pour les premiers, et plus particulièrement pour Benn Michaels, la discrimination positive est génératrice d'illusions, car elle semble confirmer qu'il existe vraiment une méritocratie (p. 84). Pour les seconds, « [1']inégalité formelle légitime l'objectif de construire une égalité réelle de résultats» (p.86). Ce livre, qui prône le principe de l'égale dignité de tous les individus, a le mérite de mettre en dialogue les recommandations duConseil de l'Europe et différents travaux académiques. Dans la conclusion, l'auteur constate que « [l]es brassages de populations au cours des générations passées ont été tels qu'il est souvent difficile de savoir où plongent les racines des arrière-grands-parents » (p. 93). Cela semble être une réponse à la question que le lecteur se pose en lisant ce texte: à quel moment l'immigré devient-il autochtone ? Mais si « [l]e passé est ainsi fait de cette diversité » (ibid.), le fait de ne plus garder en mémoire l'origine et les spécificités culturelles et linguistiques de ses propres arrière-grands-parents oriente plus vers la thèse de l'assimilation que vers celle de l'interculturalité (Pour une analyse des liens entre générations dans le contexte migratoire, voir Claudine Attias-Donfut et François-Charles Wolff, Le destin des enfants d'immigrés. Un désenchaînement des générations, Paris, Stock, 2009). Dans ce cas, le brassage conduit au gommage de la diversité. Cette évolution des générations passées est-elle compatible avec les dynamiques de nos sociétés globalisées et, encore mieux, constitue-elle un modèle à imiter ? 